\title{
Effects of on- and off-pump coronary artery surgery on graft patency, survival, and health-related quality of life: Long-term follow-up of 2 randomized controlled trials
}

Gianni D. Angelini, MCh, MD, FRCS, FETCS, ${ }^{a}$ Lucy Culliford, BSc, MSc, PhD, ${ }^{a}$ David K. Smith, BSc, MRCP, ${ }^{a}$

Mark C. K. Hamilton, MRCP, FRCR, ${ }^{b}$ Gavin J. Murphy, BSc, ChB, MD, FRCS(CTh), ${ }^{a}$ Raimondo Ascione, MD, MCh, FRCS, FETCS, ${ }^{\mathrm{a}}$ Andreas Baumbach, MD, FRCP, FESC, ${ }^{\mathrm{b}}$ and Barnaby C. Reeves, BA, MSc, DPhil ${ }^{\mathrm{a}}$

Objective: Off-pump coronary artery bypass grafting reduces postoperative morbidity and uses fewer resources than conventional surgical intervention with cardiopulmonary bypass. However, only $15 \%$ to $20 \%$ of coronary artery bypass grafting operations use off-pump coronary artery bypass. One reason for not using off-pump coronary artery bypass might be the surgeon's concern about the long-term patency of grafts performed with this technique. Therefore our objective was to compare long-term outcomes in patients randomized to off-pump coronary artery bypass or coronary artery bypass grafting with cardiopulmonary bypass.

Methods: Participants in 2 randomized trials comparing off-pump coronary artery bypass and coronary artery bypass grafting with cardiopulmonary bypass were followed up for 6 to 8 years after surgical intervention to assess graft patency, major adverse cardiac-related events, and health-related quality of life. Patency was assessed by using multidetector computed tomographic coronary angiographic analysis with a 16-slice scanner. Two blinded observers classified proximal, body, and distal segments of each graft as occluded or not. Major adverse cardiac-related events and health-related quality of life were obtained from questionnaires given to participants and family practitioners.

Results: Patency was studied in 199 and health-related quality of life was studied in 299 of 349 survivors. There was no evidence of attrition bias. The likelihood of graft occlusion was no different between off-pump coronary artery bypass $(10.6 \%)$ and coronary artery bypass grafting with cardiopulmonary bypass $(11.0 \%)$ groups (odds ratio, 1.00; 95\% confidence interval, $0.55-1.81 ; P>.99)$. Graft occlusion was more likely at the distal than the proximal anastomosis (odds ratio, 1.11; 95\% confidence interval, 1.02-1.20). There were also no differences between the off-pump coronary artery bypass and coronary artery bypass grafting with cardiopulmonary bypass groups in the hazard of death (hazard ratio, 1.24; 95\% confidence interval, $0.72-2.15$ ) or major adverse cardiac-related events or death (hazard ratio, $0.84 ; 95 \%$ confidence interval, $0.58-1.24$ ), or mean health-related quality of life across a range of domains and instruments.

Conclusions: Long-term health outcomes with off-pump coronary artery bypass are similar to those with coronary artery bypass grafting with cardiopulmonary bypass when both operations are performed by experienced surgeons.

\section{Supplemental material is available online.}

\footnotetext{
From the Bristol Heart Institute, ${ }^{\text {a }}$ University of Bristol, Bristol Royal Infirmary, Bristol, United Kingdom, and United Bristol Healthcare NHS Trust, ${ }^{\mathrm{b}}$ Bristol Royal Infirmary, Bristol, United Kingdom.

The British Heart Foundation (reference PG/04/079/17372) funded this long-term follow up. The original Beating Heart Against Cardioplegic Arrest Studies 1 and 2 trials were funded by the Garfield Weston Trust, Sir Siegmund Warburg's Voluntary Settlement, and the British Heart Foundation.

Read at the Eighty-eighth Annual Meeting of The American Association for Thoracic Surgery, San Diego, Calif, May 10-14, 2008.

Received for publication March 26, 2008; revisions received Aug 19, 2008; accepted for publication Sept 19, 2008.

Address for correspondence: Gianni D. Angelini, MCh, MD, FRCS, FETCS, Bristol Heart Institute, University of Bristol, Level 7, Bristol Royal Infirmary, Bristol BS2 8HW, United Kingdom (E-mail: g.d.angelini@bristol.ac.uk).

J Thorac Cardiovasc Surg 2009;137:295-303

0022-5223/\$36.00

Copyright (c) 2009 by The American Association for Thoracic Surgery

doi:10.1016/j.jtcvs.2008.09.046
}

There is high-quality evidence that off-pump coronary artery bypass (OPCAB) grafting reduces the risk of postoperative morbidity and ${ }^{1,2}$ intensive care unit and hospital stays ${ }^{1}$ and uses fewer resources ${ }^{3-5}$ than conventional surgery with cardiopulmonary bypass (CPB). However, only a minority of coronary artery bypass grafting (CABG) operations worldwide, about $15 \%$ to $20 \%$, are carried out with the OPCAB technique. $^{6,7}$

Surgeons might be reluctant to take up OPCAB because of concerns that the technique requires distal anastomoses to be performed on the beating heart, potentially compromising long-term patency. The literature on graft patency from randomized controlled trials (RCTs) of OPCAB versus $\mathrm{CABG}-\mathrm{CPB}$ is inconsistent, and authors have reported findings for only relatively short durations of follow-up. ${ }^{1,8}$

We performed 2 of the earliest RCTs of OPCAB versus CABG-CPB: the Beating Heart Against Cardioplegic Arrest Study (BHACAS) 1 and 2. We have previously reported clinical and health-related quality of life (HRQoL) findings at 


$$
\begin{aligned}
& \text { Abbreviations and Acronyms } \\
& \begin{aligned}
\text { BHACAS }= & \text { Beating Heart Against Cardioplegic } \\
& \text { Arrest Study } \\
\text { CABG }= & \text { coronary artery bypass grafting } \\
\text { CI } & =\text { confidence interval } \\
\text { CPB } & =\text { cardiopulmonary bypass } \\
\text { HRQoL }= & \text { health-related quality of life } \\
\text { ITA } & =\text { internal thoracic artery } \\
\text { MACE } & =\text { major adverse cardiac-related event } \\
\text { MDCTA }= & \text { multidetector computed tomography } \\
& \text { coronary angiography } \\
\text { OPCAB }= & \text { off-pump coronary artery bypass } \\
\text { RCT } & =\text { randomized controlled trial } \\
\text { SD } & =\text { standard deviation } \\
\text { SV } & =\text { saphenous vein }
\end{aligned}
\end{aligned}
$$

2 to 3 years. ${ }^{9,10}$ Here our objective is to report long-term follow-up (6-8 years) in survivors for clinical outcomes, HRQoL, and graft patency by using multidetector computed tomographic coronary angiographic (MDCTA) analysis. ${ }^{11}$

\section{MATERIALS AND METHODS BHACAS Trials}

Details of the trials have been reported elsewhere. ${ }^{9,12}$ Patients were recruited from March 1997 to August 1998 (BHACAS 1) and from September 1998 to November 1999 (BHACAS 2). Participants were randomly assigned to the OPCAB or CABG-CPB groups. Ethical approval to carry out the studies was obtained from the local research ethics committee (reference E3791).

\section{MDCTA Protocol}

MDCTA is a noninvasive method of imaging with high sensitivity and specificity compared with conventional coronary angiography. ${ }^{11}$ Exclusion criteria for MDCTA were as follows: inability to lie flat, heart rate greater than 100 beats/min, allergy to contrast medium, impaired renal function (serum creatinine, $>130 \mu \mathrm{mol} / \mathrm{L}$ ), pregnancy, or inability to provide informed consent. Patients with a heart rate of greater than 65 beats/min and no contraindication to $\beta$-blockade were given 50 to $100 \mathrm{mg}$ of metoprolol 60 to 90 minutes before MDCTA.

A 16-slice scanner (Somatom Sensation 16; Siemens, Berlin, Germany) was used. The scanning protocol consisted of 3 steps. First, an initial short tomogram $(50 \mathrm{~mA} ; 80 \mathrm{kV}$; collimation, $1 \mathrm{~mm})$ was performed to set upper and lower scan levels and reconstruction margins for the heart. Second, a test bolus scan was administered to determine the circulation time of the contrast medium. The start of the contrast-enhanced coronary scan was adapted by adding 3 seconds to the calculated circulation time to allow for homogeneous mixing of the contrast medium throughout the coronary arterial tree. Third, a contrast MDCTA spiral scan $(550 \mathrm{mAs}, 120 \mathrm{kV}, 16$ $\times 0.75-\mathrm{mm}$ slice width collimation, 0.42 -second rotation time, and 2.8 $\mathrm{mm}$ feed per rotation) was performed with contrast medium $(125 \mathrm{~mL}$ of Iomeron 400 at $4 \mathrm{~mL} / \mathrm{s}$, Braceo Group, San Donato Milanese, Italy) infused at the start time, as described above. The scan was in a caudocranial direction, with the upper margin at the level of the clavicles to include internal thoracic artery (ITA) grafts and the lower margin just below the base of the heart.

Dedicated spiral algorithms provided 105- to 250-ms temporal resolution from retrospective electrocardiogram-triggered phase reconstruction, depending on heart rate and the vessel segment under consideration. The coronary scan has serial phase image reconstruction performed with retrospective gating between $-60 \%$ and $-40 \%$ absolute reverse (1-mm slice width, B30f medium smooth kernel).

\section{Assessment of MDCTA Images}

Images were interpreted on computer workstations (Wizard/Leonardo, Siemens) by 2 independent observers blinded to randomized allocation. Analysis of grafts was carried out from thin maximum-intensity projection slices and 3-dimensional volume images. Scans were reported by using an established system. ${ }^{13}$

Each graft was classified by conduit type (pedicle ITA or gastroepiploic artery [1 graft only], free ITA, radial artery, and saphenous vein [SV]) and assessed in 3 segments: the proximal anastomosis, the body of the graft, and the distal anastomosis. Each segment was classified as patent (flow visible), occluded, or not analyzable (eg, because the segment was obscured by a metal ligature clip or because the image quality was poor).

\section{Follow-up to Assess Outcomes}

Participants were followed up through the National Health Service Strategic Tracing Service and by annual questionnaire for major adverse cardiac-related events (MACEs); we also sent annual questionnaires to family practitioners. ${ }^{14}$ MACEs were defined as follows: (1) recurrent angina (hospital visit for angina reported by patient or hospital admission for angina reported by general practitioner); (2) myocardial infarction (hospital visit for myocardial infarction reported by patient or hospital admission for myocardial infarction reported by general practitioner); and (3) repeat revascularization (repeat operation or angioplasty since the index operation reported by patient or general practitioner). Reported admissions to the Bristol Heart Institute were all verified.

Survivors were sent $4 \mathrm{HRQoL}$ questionnaires ${ }^{10}$ (ie, the Seattle Angina Questionnaire, ${ }^{15}$ the Coronary Revascularisation Outcome Questionnaire, ${ }^{16}$ the Short-Form Health Survey 36 version $2,{ }^{17,18}$ and the EuroQol ${ }^{19}$ ) with their annual surveillance questionnaire. If there was no response to the first mailing, after a month, a second questionnaire was sent. If neither elicited a response, the patient was telephoned to find out whether there was a particular reason, such as ill health, preventing completion of forms. Patients who indicated that they would like to undergo MDCTA but did not return their questionnaires were asked to complete them when attending for MDCTA. Patients who were not able to complete the questionnaires themselves (eg, because of poor sight) were invited to complete them by telephone or face-to-face.

\section{Statistical Analyses}

Patency. Agreement between initial assessments of graft patency by the 2 observers was described by using the $\kappa$ statistic. When grafts were assessed by observer 1 but classified as not analyzable by observer 2 , the assessment of observer 1 was used and vice versa. Disagreements between observers were reconciled, if necessary, by a third observer.

Patency was analyzed by means of multivariable logistic regression (occluded or not), with segments as individual observations. Robust standard errors were estimated to take account of nesting of grafts within patients. Segments that could not be analyzed by either observer were excluded. Randomized allocation, trial (BHACAS 1 or 2), segment, conduit, and graft territory were included as covariates. Two interactions with operation type were also investigated: (1) allocation by trial to check the validity of pooling data across trials and (2) allocation by segment to test the prior hypothesis that distal anastomoses are more likely to occlude with OPCAB than with CABG-CPB.

All-cause mortality and MACEs. Cox regression analyses were carried out for 3 outcomes: (1) all-cause mortality; (2) all-cause mortality or MACEs; and (3) MACEs only. All participants were included in the analysis of all-cause mortality, censoring surviving participants at the last known date of follow-up. For survival free from MACEs or death, participants were 
censored at the last known date of follow-up if alive and free from MACEs. For the analysis of MACEs only, participants were censored at death if free from a MACE at this time. Models included type of operation and trial (ie, BHACAS 1 or 2). The interaction of allocation by trial was tested. Validity of the proportional hazards assumption was checked in all models.

HRQoL. HRQoL questionnaires were scored as previously reported. ${ }^{10}$ For all dimensions, higher scores represent better HRQoL. Linear regression models fitted type of operation and trial. The interaction of operation allocation by trial was tested. Confidence intervals (CIs) for differences between the CABG-CPB and OPCAB groups were estimated by means of bootstrapping because some distributions of scores were skewed. Analyses of all outcomes were by intention to treat.

Sample size justification. Original sample sizes were based on length of stay. ${ }^{9}$ In the protocol for this follow-up, we stated that patency would be analyzed for grafts and not patients, gaining additional power from multiple grafts per patient (approximately 2.5 grafts per patient). We expected to analyze data for 320 patients, providing 800 grafts for study. Assuming a design effect of 1.3 (intraclass correlation, 0.2 ), the study would have an effective sample size of about 610 "independent" observations.

The graft occlusion rate was expected to vary by conduit: in patients undergoing $\mathrm{CABG}-\mathrm{CPB}$, we expected approximately $10 \%$ of arterial and approximately $40 \%$ of SV grafts to be occluded. (Although the occlusion rate was expected to vary by conduit type, no interaction of conduit type and operation type was hypothesized.) Among participants who contributed to the previous follow-up, ${ }^{10} 47 \%$ (388/834) of grafts were arterial conduits. Therefore the overall graft "failure" rate in the CABG-CPB group was estimated to be approximately $26 \%$ : $(10 \% \times 47 \%)+(40 \% \times 53 \%)$.

Based on the above assumptions, we calculated that the study would have $80 \%$ power to detect an absolute difference of $11 \%$ (ie, $26 \%$ vs $37 \%$ or a relative risk of 1.4$)$ in the risk of graft occlusion $(\alpha=.05,2$-tailed) or to exclude the possibility of differences smaller than $8 \%(\alpha=.05,2$ tailed). The pooled data had $90 \%$ power to detect a small-to-moderate standardized difference (0.43) in HRQoL ( $\alpha=.01,2$-tailed), assuming about $90 \%$ of survivors $(350 \times 0.90 \approx 320)$ responded.

\section{RESULTS}

Deaths, numbers and reasons for loss to follow-up, and numbers contributing to analyses are shown in Figure 1. Fifty-two $(13.0 \%)$ of 401 randomized participants had died, 28 in the OPCAB group (14\%) and 24 in the CABG-CPB group $(12 \%)$; of the remaining 349, $299(86 \%)$ completed HRQoL questionnaires, and $199(57 \%)$ had MDCTA scans.

Table 1 shows the baseline characteristics of all survivors, those who attended for MDCTA, and those who completed HRQoL questionnaires. Survivors had similar characteristics at baseline to the entire randomized cohort'; only the proportions with unstable angina and Parsonnet scores of greater than 10 appeared to be lower among survivors, equally for both the CABG-CPB and OPCAB groups. Compared with all survivors, those who had MDCTA scans tended to be slightly younger and were more likely to be male but otherwise had similar characteristics. Baseline characteristics of participants who completed HRQoL questionnaires were similar to those for all survivors and similar for the $\mathrm{CABG}-\mathrm{CPB}$ and $\mathrm{OPCAB}$ groups.

\section{Graft Patency}

A total of 505 grafts were studied in 199 participants between January 31, 2005, and August 3, 2006. At least 1 graft

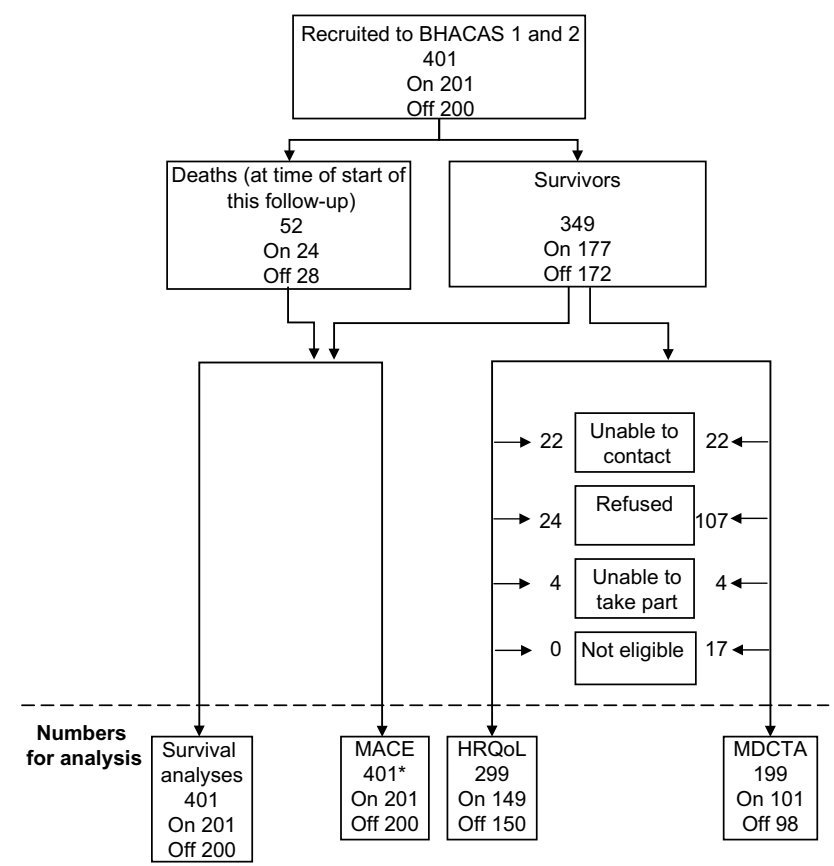

FIGURE 1. Flow diagram showing the numbers of participants randomized in the Beating Heart Against Cardioplegic Arrest Studies surviving to this follow-up, with data contributing to analyses of different outcomes (and reasons for data not being available). MACE, Major adverse cardiac event; $H R Q O L$, health-related quality of life; MDCTA, multidetector computed tomography coronary angiography.

was analyzable in each participant, but both observers could not analyze $13(2.6 \%)$ grafts, and one or the other observer could not analyze another $24(4.8 \%)$ grafts. Mean durations of follow-up from the operation to MDCTA were 85.1 months (standard deviation [SD], 4.8) and 85.8 months ( $\mathrm{SD}, 4.7$ ) for the $\mathrm{CABG}-\mathrm{CPB}$ and OPCAB groups, respectively. Conduits and coronary territories grafted were similar in the 2 groups (Table 2).

There was moderate agreement between initial assessments of the 2 observers $(\kappa=0.62$ for 505 grafts, analyzing grafts classified as not analyzable in a separate category; $\kappa=$ 0.71 for 468 grafts classified by both observers as occluded or not). Overall, 53 (10.8\%) of 492 grafts were classified as occluded after disagreements were reconciled. Percentages of grafts classified as occluded were similar in the CABGCPB and OPCAB groups, both overall (27/255 [10.6\%] and 26/237 [11.0\%], respectively; Table 3) and for arterial and SV grafts separately (arterial grafts, 13/126 [10.3\%] vs $8 / 116$ [6.9\%]; SV grafts, $14 / 129$ [10.9\%] vs $18 / 121$ [14.9\%], respectively). SV grafts were more likely to be occluded (32/250 [12.8\%]) than arterial grafts (21/242 [8.7\%]) but not to the extent expected at the outset.

The logistic regression analysis of graft occlusion showed no evidence that grafts were more likely to be occluded in OPCAB than in CABG-CPB participants (odds ratio, 1.00; $95 \%$ CI, $0.55-1.81 ; P>.99$; model details in are shown in 
TABLE 1. Baseline demographic and clinical characteristics of CABG-CPB and OPCAB survivors

\begin{tabular}{|c|c|c|c|c|c|c|c|c|c|c|c|c|}
\hline \multirow[b]{3}{*}{ Variable } & \multicolumn{4}{|c|}{ All survivors } & \multicolumn{4}{|c|}{ Patients who had MDCTA } & \multicolumn{4}{|c|}{ Patients returning HRQoL questionnaires } \\
\hline & \multicolumn{2}{|c|}{$\begin{array}{c}\text { CABG-CPB } \\
(\mathbf{n}=\mathbf{1 7 7}) \\
\end{array}$} & \multicolumn{2}{|c|}{$\begin{array}{c}\text { OPCAB } \\
(\mathbf{n}=\mathbf{1 7 2}) \\
\end{array}$} & \multicolumn{2}{|c|}{$\begin{array}{c}\text { CABG-CPB } \\
(\mathbf{n}=\mathbf{1 0 1}) \\
\end{array}$} & \multicolumn{2}{|c|}{$\begin{array}{l}\text { OPCAB } \\
(n=98) \\
\end{array}$} & \multicolumn{2}{|c|}{$\begin{array}{c}\text { CABG-CPB } \\
(n=149)\end{array}$} & \multicolumn{2}{|c|}{$\begin{array}{c}\text { OPCAB } \\
(n=150)\end{array}$} \\
\hline & No. & $\%$ & No. & $\%$ & No. & $\%$ & No. & $\%$ & No. & $\%$ & No. & $\%$ \\
\hline BHACAS 2 & 89 & 50.3 & 88 & 51.2 & 52 & 51.5 & 48 & 49.0 & 71 & 47.7 & 77 & 51.3 \\
\hline Male sex & 147 & 83.1 & 143 & 83.1 & 89 & 88.1 & 87 & 88.8 & 129 & 86.6 & 127 & 84.7 \\
\hline Age $(y)^{*}$ & \multicolumn{2}{|c|}{$60.5 \pm 8.6$} & \multicolumn{2}{|c|}{$62.0 \pm 8.7$} & \multicolumn{2}{|c|}{$59.1 \pm 8.3$} & \multicolumn{2}{|c|}{$61.0 \pm 8.3$} & \multicolumn{2}{|c|}{$60.9 \pm 8.5$} & \multicolumn{2}{|c|}{$62.1 \pm 8.2$} \\
\hline Diabetes & 41 & 23.2 & 42 & 24.4 & 22 & 21.8 & 22 & 22.5 & 34 & 22.8 & 37 & 24.7 \\
\hline \multicolumn{13}{|c|}{ Previous myocardial infarction } \\
\hline$>1 \mathrm{mo}$ & 61 & 34.5 & 65 & 37.8 & 40 & 39.6 & 34 & 34.7 & 52 & 34.9 & 56 & 37.3 \\
\hline$<1 \mathrm{mo}$ & 11 & 6.2 & 14 & 8.1 & 5 & 5.0 & 5 & 5.1 & 9 & 6.0 & 12 & 8.0 \\
\hline Hypertension & 87 & 49.2 & 102 & 59.3 & 51 & 50.5 & 62 & 63.3 & 76 & $51.0 \dagger$ & 94 & $62.7 \dagger$ \\
\hline Hypercholesterolemia & 126 & 71.2 & 134 & 77.9 & 72 & 71.3 & 77 & 78.6 & 107 & 71.8 & 116 & 77.3 \\
\hline \multicolumn{13}{|l|}{ Smoking history } \\
\hline Current & 20 & 11.3 & 17 & 9.9 & 10 & 9.9 & 9 & 9.2 & 13 & 8.7 & 11 & 7.3 \\
\hline Exsmoker & 106 & 59.9 & 108 & 62.8 & 56 & 55.5 & 64 & 65.3 & 90 & 60.4 & 96 & 64.0 \\
\hline Nonsmoker & 51 & 28.8 & 47 & 27.3 & 35 & 34.7 & 25 & 25.5 & 46 & 30.9 & 43 & 28.8 \\
\hline Unstable angina & 62 & 35.0 & 65 & 37.8 & 33 & 32.7 & 36 & 36.7 & 46 & 30.9 & 57 & 38.0 \\
\hline \multicolumn{13}{|l|}{ Angina class (CCS) } \\
\hline I & 22 & 12.4 & 15 & 8.7 & 14 & 13.9 & 6 & 6.1 & 21 & 14.1 & 12 & 8.0 \\
\hline II & 62 & 35.0 & 60 & 34.9 & 32 & 31.7 & 34 & 34.7 & 58 & 38.9 & 53 & 35.3 \\
\hline III & 54 & 30.5 & 54 & 31.4 & 32 & 31.7 & 32 & 32.7 & 43 & 28.9 & 47 & 31.3 \\
\hline IV & 39 & 22.0 & 43 & 25.0 & 23 & 22.8 & 26 & 26.5 & 27 & 18.1 & 38 & 25.3 \\
\hline Ejection fraction $<50 \%$ & 41 & 23.2 & 37 & 21.5 & 25 & 24.8 & 16 & 16.3 & 35 & 23.5 & 32 & 21.3 \\
\hline Parsonnet score $>10$ & 20 & 11.3 & 28 & 16.3 & 9 & 8.9 & 10 & 10.2 & 18 & 12.1 & 23 & 15.3 \\
\hline \multicolumn{13}{|l|}{ No. of distal anastomoses } \\
\hline 1 & 17 & 9.6 & 16 & 9.3 & 8 & 7.9 & 10 & 10.2 & 13 & 8.7 & 14 & 9.3 \\
\hline 2 & 60 & 33.9 & 74 & 43.0 & 36 & 35.6 & 44 & 44.9 & 53 & 35.6 & 63 & 42.0 \\
\hline 3 & 76 & 42.9 & 65 & 37.8 & 44 & 43.6 & 33 & 33.7 & 62 & 41.6 & 56 & 37.3 \\
\hline$\geq 4$ & 24 & 13.6 & 17 & 9.9 & 13 & 12.9 & 11 & 11.2 & 21 & 14.1 & 17 & 11.3 \\
\hline
\end{tabular}

$M D C T A$, Multidetector computed tomography coronary angiography; $H R Q L L$, health-related quality of life; $C A B G-C P B$, coronary artery bypass grafting with cardiopulmonary bypass; $O P C A B$, off-pump coronary artery bypass; BHACAS, Beating Heart Against Cardioplegic Arrest Study; CCS, Canadian Cardiovascular Society. *Reported as the mean and standard deviation. $\dagger$ The difference between the coronary artery bypass grafting with cardiopulmonary bypass and off-pump coronary artery bypass groups was statistically significant $(P=.04)$. No other differences for all survivors, patients who had multidetector computed tomographic coronary angiography, or patients who returned health-related quality of life questionnaires were significant.

Table E1). Grafts carried out in BHACAS 2 were less likely to be occluded (odds ratio, $0.49 ; 95 \% \mathrm{CI}, 0.27-0.89 ; P=$ .02). Graft occlusion was more likely at the distal than the proximal anastomosis (odds ratio, $1.11 ; 95 \% \mathrm{CI}, 1.02-$ $1.20 ; P=.02)$; the graft body was no more likely to be occluded than the proximal anastomosis (odds ratio, 1.02;
95\% CI, 0.92-1.14; $P=.69)$. The interaction of operation allocation by trial was not significant $(P=.59)$ but suggested occlusion was more likely with OPCAB compared with CABG-CPB in BHACAS 1 and vice versa in BHACAS 2 (see Table E2 and Figure E1). The interaction of operation type and graft segment reached statistical significance

TABLE 2. Distribution of 505 grafts by conduit, coronary territory, and operation type in CABG-CPB and OPCAB 199 patients who had MDCTA scans

\begin{tabular}{|c|c|c|c|c|c|c|}
\hline \multirow[b]{3}{*}{ Conduit } & \multicolumn{3}{|c|}{ CABG-CPB } & \multicolumn{3}{|c|}{ ОРСАВ } \\
\hline & \multicolumn{3}{|c|}{ Coronary territory } & \multicolumn{3}{|c|}{ Coronary territory } \\
\hline & Anterior & Lateral & Inferior & Anterior & Lateral & Inferior \\
\hline Pedicle internal thoracic artery & $103(39.0 \%)$ & $10(3.8 \%)$ & $7(2.7 \%)$ & $89(36.9 \%)$ & $4(1.7 \%)$ & $9(3.7 \%)$ \\
\hline Free internal thoracic artery & $0(0.0 \%)$ & $3(1.1 \%)$ & $0(0.0 \%)$ & $4(1.7 \%)$ & $0(0.0 \%)$ & $0(0.0 \%)$ \\
\hline Radial artery & $1(0.4 \%)$ & $3(1.1 \%)$ & $6(2.3 \%)$ & $2(0.8 \%)$ & $5(2.1 \%)$ & $6(2.5 \%)$ \\
\hline Saphenous vein & $27(10.2 \%)$ & $50(18.9 \%)$ & $54(20.5 \%)$ & $39(16.2 \%)$ & $32(13.3 \%)$ & $51(21.2 \%)$ \\
\hline Total & $131(49.6 \%)$ & $66(25.0 \%)$ & $67(25.4 \%)$ & $134(55.6 \%)$ & $41(17.0 \%)$ & $66(27.4 \%)$ \\
\hline
\end{tabular}


TABLE 3. Classification of patency* by type of conduit in 492 grafts in 101 patients undergoing CABG-CPB and 98 patients undergoing OPCAB

\begin{tabular}{|c|c|c|c|c|}
\hline \multirow[b]{2}{*}{ Conduit } & \multicolumn{2}{|c|}{ CABG-CPB } & \multicolumn{2}{|c|}{ OPCAB } \\
\hline & $\begin{array}{c}\text { Total } \\
\text { analyzed } \dagger\end{array}$ & $\begin{array}{l}\text { Occluded, } \\
\text { n }(\%)\end{array}$ & $\begin{array}{c}\text { Total } \\
\text { analyzed } \dagger\end{array}$ & $\begin{array}{c}\text { Occluded, } \\
\text { n }(\%) \\
\end{array}$ \\
\hline $\begin{array}{l}\text { Pedicle internal thoracic } \\
\text { artery }\end{array}$ & 114 & $12(10.5)$ & 101 & $6(5.9)$ \\
\hline $\begin{array}{l}\text { Free internal thoracic } \\
\text { artery }\end{array}$ & 3 & $0(0.0)$ & 4 & $0(0.0)$ \\
\hline Radial artery & 9 & $1(11.1)$ & 11 & $2(18.2)$ \\
\hline Saphenous vein & 129 & $14(10.9)$ & 121 & $18(14.9)$ \\
\hline Total & 255 & 27 (10.6) & 237 & $26(11.0)$ \\
\hline
\end{tabular}

$C A B G-C P B$, Coronary artery bypass grafting with cardiopulmonary bypass; $O P C A B$, off-pump coronary artery bypass. *Grafts were classified as occluded if any segment was occluded. †In addition, 9 grafts in patients undergoing coronary artery bypass grafting with cardiopulmonary bypass and 4 grafts in patients undergoing off-pump coronary artery bypass could not be analyzed; 7 ( 6 and 1 in the coronary artery bypass grafting with cardiopulmonary bypass/off-pump coronary artery bypass groups, respectively) were pedicle internal thoracic artery grafts, 3 were radial artery ( 1 and 2, respectively) grafts, and 3 were saphenous vein ( 2 and 1 , respectively) grafts.

$(P=.04)$ but was not included in the final model because it did not support the prior hypothesis that distal anastomoses performed with $\mathrm{OPCAB}$ were more likely to be occluded; odds ratios for OPCAB versus $\mathrm{CABG}-\mathrm{CPB}$ were 1.08 , 1.05 , and 0.88 for proximal, body, and distal segments, respectively (see Table E3 and Figure E2).

\section{Survival and MACEs}

Mean durations of follow-up for survival were 75.5 (SD, 20.6) and 76.7 (SD, 19.3) months for OPCAB and CABGCPB participants; there were 23 and 29 deaths in each group, respectively. Cox regression showed no difference in survival between the 2 groups (hazard ratio, 1.24; $95 \%$ CI, $0.72-2.15 ; P=.44)$. There was no effect of trial $(P=.51)$ or the interaction of operation allocation by trial $(P=.36)$.
MACEs that occurred in the 2 groups are shown in Table 4. There were 49 and 39 MACEs in the CABG-CPB and OPCAB groups and a further 7 and 10 deaths as first events in each group, respectively (105 participants with $\geq 1$ event). There are no apparent differences in the number or nature of first-reported MACEs between the $\mathrm{CABG}-\mathrm{CPB}$ and OPCAB groups.

Mean duration of follow-up in analyses of survival free from MACEs or death was 62.7 (SD, 29.4) and 64.8 (SD, 28.9) months for the CABG-CPB and OPCAB participants, respectively. Cox regression showed no difference between the 2 groups (hazard ratio, $0.84 ; 95 \% \mathrm{CI}, 0.58-1.24 ; P=$ .39 ; Figure 2$)$. There were no effects of trial $(P=.73)$ or the interaction of operation allocation by trial $(P=.31)$. Survival free from MACEs or death at 5 years was estimated to be $78.7 \%(72.2 \%-83.8 \%)$ and $81.8 \%(75.6 \%-86.6 \%)$, respectively, in the $\mathrm{CABG}-\mathrm{CPB}$ and OPCAB groups. Similarly, there was no difference in survival free from MACEs only between the 2 groups (hazard ratio, $0.77 ; 95 \% \mathrm{CI}$, $0.51-1.18 ; P=.24)$, with no effects of trial $(P=.15)$ or the interaction of operation allocation by trial $(P=.21)$.

\section{HRQoL}

Mean durations of follow-up among respondents to the HRQoL questionnaires were 83.6 (SD, 4.9) and 83.1 (SD, 5.7) months for the $\mathrm{CABG}-\mathrm{CPB}$ and $\mathrm{OPCAB}$ groups, respectively. Table 5 shows the mean scores for different domains of HRQoL for the 4 instruments by operation type and differences between the group means. None of the differences were statistically significant.

\section{DISCUSSION}

\section{Main Findings}

The key findings from the long-term follow-up of participants in the BHACAS 1 and 2 trials are that there were no differences between the $\mathrm{CABG}-\mathrm{CPB}$ and $\mathrm{OPCAB}$ groups in

TABLE 4. First major adverse cardiac-related event experienced and deaths by allocation and time period

\begin{tabular}{|c|c|c|c|c|c|c|}
\hline \multirow[b]{2}{*}{ Event } & \multicolumn{3}{|c|}{ CABG-CPB $(n=201)$} & \multicolumn{3}{|c|}{ OPCAB $(n=200)$} \\
\hline & $\leq \mathbf{1} \mathbf{y}$ & $>1$ and $\leq 4 \mathrm{y}$ & $>4 y$ & $\leq \mathbf{1} \mathbf{y}$ & $>1$ and $\leq 4 \mathrm{y}$ & $>4 y$ \\
\hline Angina* & $16(8.0 \%)$ & $14(7.0 \%)$ & $8(4.0 \%)$ & $15(7.5 \%)$ & $10(5.0 \%)$ & $3(1.5 \%)$ \\
\hline Myocardial infarction & $3(1.5 \%)$ & $2(1.0 \%)$ & $3(1.5 \%)$ & $2(1.0 \%)$ & $2(1.0 \%)$ & $4(2.0 \%)$ \\
\hline Revascularization $\dagger$ & $0(0.0 \%)$ & $1(0.5 \%)$ & $2(1.0 \%)$ & $2(1.0 \%)$ & $0(0.0 \%)$ & $1(0.5 \%)$ \\
\hline Death (as first event) & $0(0.0 \%)$ & $0(0.0 \%)$ & $7(3.5 \%)$ & $0(0.0 \%)$ & $1(0.5 \%)$ & $9(4.5 \%)$ \\
\hline Death (not as first event) $\ddagger$ & $3(1.5 \%)$ & $10(5.0 \%)$ & $3(1.5 \%)$ & $2(1.0 \%)$ & $6(3.0 \%)$ & $11(5.5 \%)$ \\
\hline Any death $\S$ & $3(1.5 \%)$ & $10(5.0 \%)$ & $10(5.0 \%)$ & $2(1.0 \%)$ & $7(3.5 \%)$ & $20(10.0 \%)$ \\
\hline
\end{tabular}

Percentages are expressed with respect to denominators of 201 and 200 participants who had coronary artery bypass grafting with cardiopulmonary bypass and off-pump coronary artery bypass, respectively. Note that percentages for the last 2 rows are distinct from the first 4 rows. $C A B G-C P B$, Coronary artery bypass grafting with cardiopulmonary bypass; $O P C A B$, off-pump coronary artery bypass. *Eight patients who reported angina subsequently had a myocardial infarction, of whom 3 also reported revascularization (all were in the coronary artery bypass grafting with cardiopulmonary bypass group; the dates of myocardial infarction and revascularization were the same for 2 patients, and the date of myocardial infarction preceded revascularization for the third patient). $\dagger$ All 6 patients who underwent revascularization as first events had percutaneous coronary intervention. A further 9 patients are known to have had revascularization after recurrence of angina or myocardial infarction; 7 had percutaneous coronary intervention, and 2 had repeat operations. $\ddagger$ Numbers in this row represent deaths in participants with up-to-date annual surveillance at the time of death (ie, deaths in patients included in the analysis of survival free from major adverse cardiac event events but that were not included as events because they were preceded by a major adverse cardiac event). $\S \mathrm{Numbers} \mathrm{in} \mathrm{this} \mathrm{row} \mathrm{represent}$ all deaths. 
Cardiac event free survival including death

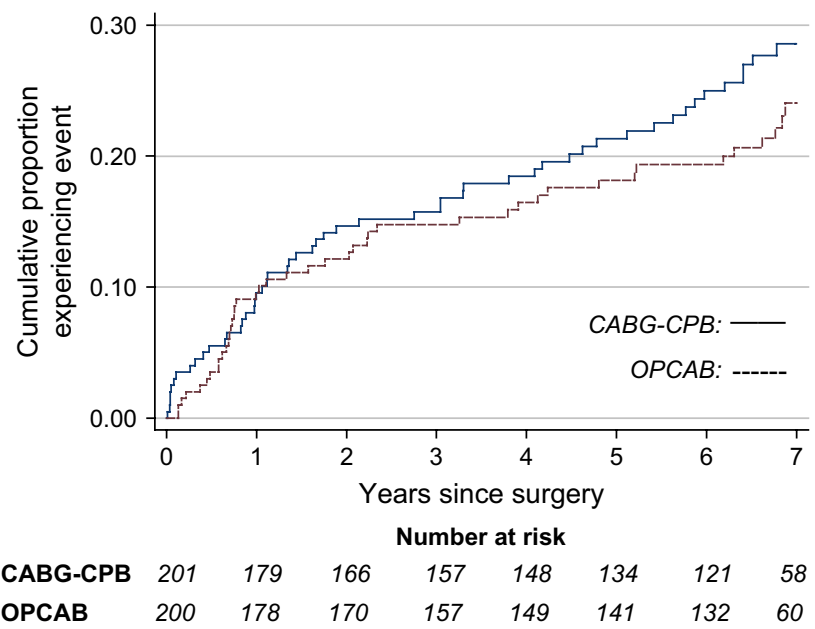

FIGURE 2. Kaplan-Meier graph showing cardiac event-free survival, including death. $C A B G-C P B$, Coronary artery bypass grafting with cardiopulmonary bypass; $O P C A B$, off-pump coronary artery bypass.

graft patency, survival and survival free from MACEs, and HRQoL 6 to 8 years after surgical intervention.

\section{Strengths and Limitations}

Randomized allocation, which minimizes selection bias, is an important strength of the BHACAS trials which are reported here in compliance with the CONSORT statement (www.consort-statement.org). A further strength is the high proportion of screened patients who were recruited (almost $50 \%),{ }^{9}$ enhancing the applicability of the findings in our center.

Attrition during the 6 to 8 years of follow-up is the main limitation, but this does not appear to have introduced bias (ie, there was no selective attrition by group). Attrition varied by outcome. Despite its relative noninvasiveness compared with conventional angiographic analysis, only $57 \%$ of survivors underwent MDCTA. The main reason for not undergoing MDCTA was because some participants declined to be reinvestigated. However, there was no difference in the percentages of participants undergoing MDCTA or differences in their baseline characteristics by surgical group. There was less attrition with HRQoL. In survival analyses all participants contributed up to the last known follow-up. The fact that attrition bias was not observed for other outcomes suggests that informative censoring is unlikely to have affected these analyses.

Because patency data were obtained for only 492 grafts (effective sample size, 378), analyses of graft patency had $80 \%$ power to detect a relative risk of occlusion of about 2.0 (ie, $11 \%$ for the CABG-CPB group vs $22 \%$ for the OPCAB group) compared with 1.4 as proposed at the outset

TABLE 5. Mean scores and differences between the CABG-CPB and OPCAB groups for the Short-Form Health Survey 36 (SF-36), the Coronary Revascularisation Outcome Questionnaire (CROQ), the Seattle Angina Questionnaire (SAQ), and EuroQol

\begin{tabular}{|c|c|c|c|c|c|c|c|c|c|}
\hline & \multicolumn{3}{|c|}{ CABG-CPB } & \multicolumn{3}{|c|}{ ОРСАВ } & \multicolumn{3}{|c|}{ OPCAB vs CABG-CPB } \\
\hline & No. & Mean & SD & No. & Mean & SD & Difference & 95\% CI & $P$ value \\
\hline \multicolumn{10}{|l|}{$\mathrm{SF}-36^{*}$} \\
\hline PCS* & 130 & 53.4 & 9.5 & 138 & 53.0 & 8.4 & -0.37 & -2.56 to 1.82 & .74 \\
\hline MCS* & 130 & 52.4 & 9.9 & 138 & 51.0 & 7.6 & -1.39 & -3.45 to 0.66 & .18 \\
\hline \multicolumn{10}{|l|}{ CROQ } \\
\hline Core total $*$ & 148 & 49.6 & 7.5 & 145 & 50.3 & 7.0 & 0.79 & -0.87 to 2.51 & .35 \\
\hline Symptoms $\dagger$ & 146 & 85.2 & 20.0 & 144 & 86.6 & 18.6 & 1.43 & -2.81 to 5.68 & .51 \\
\hline Physical functioning $\dagger$ & 146 & 74.2 & 29.1 & 144 & 77.0 & 27.0 & 2.88 & -3.55 to 9.32 & .38 \\
\hline Cognitive functioning $\dagger$ & 147 & 78.8 & 23.3 & 146 & 81.8 & 22.7 & 3.06 & -2.08 to 8.19 & .24 \\
\hline Psychosocial functioning $\dagger$ & 148 & 82.8 & 20.9 & 146 & 85.3 & 19.0 & 2.55 & -1.83 to 6.94 & .25 \\
\hline Satisfaction $\dagger$ & 147 & 82.7 & 21.7 & 145 & 84.6 & 18.8 & 1.84 & -2.84 to 6.52 & .44 \\
\hline Adverse effects $\dagger$ & 146 & 93.5 & 11.0 & 140 & 95.3 & 8.5 & 1.86 & -0.50 to 4.23 & .12 \\
\hline \multicolumn{10}{|l|}{ SAQ } \\
\hline Physical function $†$ & 136 & 72.4 & 25.8 & 130 & 75.0 & 24.9 & 2.59 & -3.53 to 8.70 & .41 \\
\hline Angina frequency $\dagger$ & 139 & 88.8 & 22.5 & 138 & 89.6 & 19.7 & 0.76 & -4.14 to 5.65 & .76 \\
\hline Angina stability $\dagger \ddagger$ & 40 & 45.6 & 23.9 & 43 & 46.5 & 24.1 & 0.93 & -9.60 to 11.5 & .86 \\
\hline Treatment satisfaction $\dagger$ & 106 & 89.2 & 20.2 & 110 & 91.5 & 16.3 & 2.33 & -2.59 to 7.25 & .35 \\
\hline Quality of life $†$ & 117 & 74.0 & 25.7 & 118 & 77.0 & 23.4 & 3.04 & -3.38 to 9.45 & .35 \\
\hline \multicolumn{10}{|l|}{ EuroQol } \\
\hline Utility§ & 135 & 0.80 & 0.25 & 136 & 0.82 & 0.24 & 0.01 & -0.05 to 0.07 & .64 \\
\hline Health status $\S$ & 136 & 73.0 & 18.6 & 140 & 75.8 & 17.1 & 2.77 & -1.41 to 6.96 & .19 \\
\hline
\end{tabular}

Higher scores indicate better quality of life. Numbers vary because some respondents did not complete all items. $C A B G-C P B$, Coronary artery bypass grafting with cardiopulmonary bypass; $O P C A B$, off-pump coronary artery bypass. *Scored on a scale with a mean of 50 and SD of 10 . SF-36 physical (PCS) and mental (MCS) component summary scores are also normalized: 50 represents the mean for the reference (ie, "normal”) population. $\dagger$ Scored on a scale from 0 to 100 . $\ddagger$ Ninety-four participants in the coronary artery bypass grafting with cardiopulmonary bypass group and 92 in the off-pump coronary artery bypass group reported no angina; these responses do not equate to a score. $\S$ Scored on a scale from 0 to 1 . 
(ie, $26 \%$ for the $\mathrm{CABG}-\mathrm{CPB}$ group vs $37 \%$ for the OPCAB group). Note that this post-hoc calculation is based on the observed lower frequency of graft occlusion $(11 \%)$, as well as the smaller sample size available.

Finally, it is important to remember that the BHACAS trials were carried out in a single center by a single academic surgical team. This surgical team has documented innovations in OPCAB technique, their performance and that of residents learning $\mathrm{OPCAB}$, and other aspects of their experience with $\mathrm{OPCAB}$ over more than a decade. Therefore caution should be exercised in generalizing these findings to other surgeons and centers.

\section{Findings in the Context of Other Literature}

We did not synthesize our findings with those of other RCTs that have reported graft patency because the followup reported here was much longer and patency was assessed by means of MDCTA and not conventional angiographic analysis. Angiographic findings up to 1 year from previous RCTs have been pooled in a meta-analysis without statistical evidence of heterogeneity. ${ }^{8}$ However, this analysis took no account of varying duration of follow-up (from discharge to 1 year), varying attrition, or the lack of independence of grafts within patients. If occluded grafts tend to be in the same patients, as suggested by our data and by other studies, this latter failing could seriously undermine statistical inferences.

Three trials have reported 1-year patency findings that were subject to attrition, ranging from $22 \%$ to $36 \%$, and none found a significant difference. ${ }^{4,5,20}$ Two trials compared the proportion of grafts that were patent but took no account of nesting of grafts within patients; both found a difference in patency of $2 \%$ favoring the CABG-CPB group. ${ }^{4,5}$ The third trial correctly compared the proportion of patients with none, 1 , or at least 2 occluded grafts, observing differences of $2 \%$ to $6 \%$ favoring the OPCAB group. ${ }^{20}$ However, only about $50 \%$ of patients had all grafts patent at 1 year compared with $75 \%$ after 6 to 8 years in this study. One additional small trial reported that grafts performed during OPCAB were more likely to be occluded at 3 months $(88 \%$ vs $98 \%))^{21}$ This trial was not powered to find a difference in graft patency and took no account of nesting of grafts within patients.

Given the time since surgical intervention, we observed few occluded grafts. The low rate of occlusion of SV grafts was particularly surprising. This might, in part, be due to early routine administration of aspirin (300 $\mathrm{mg}$ per rectum) during the first 6 hours and lifetime use of statins after surgical intervention. Our patency findings cannot be directly compared with previous findings, not least because of potential differences between conventional angiographic analysis and MDCTA. Although MDCTA has high sensitivity and specificity in detecting graft patency, ${ }^{11}$ we cannot rule out the presence of stenoses in patent SV grafts.
Metal ligature clips prevented assessment of some ITA and radial grafts. Some arterial grafts had a thread-like appearance because of new stenosis in the native vessel or poor runoff; these were identified with difficulty, and some might have been classed as occluded. Despite these difficulties, MDCTA assessment was not biased because the radiologic assessors were blinded to randomized allocation.

Two other RCTs have assessed HRQoL, reporting that improvements in HRQoL up to 1 year were similar with CABG-CPB and OPCAB. ${ }^{4,22}$ Three-year follow-up of the BHACASs also found no difference. ${ }^{10}$ The findings reported here are consistent with these reports. We assessed patency to address particular mechanistic hypotheses about OPCAB but maintain that HRQoL provides the most important evidence about the relative effectiveness of CABG-CPB and OPCAB (ie, the patient's view).$^{10}$ Symptoms of ischemia after surgical intervention must be presumed to arise from stenoses that have developed since the operation and are likely to precede events attributable to coronary disease. Although new stenoses can arise either in grafts or native vessels, any difference in "average" symptoms between groups can be confidently attributed to operation type when patients have been randomized and there is little attrition. Also, HRQoL instruments are completely noninvasive and yield continuous scores rather than binary outcomes, resulting in greater power for a given sample size.

In addition to concern about graft patency, surgeons who use $\mathrm{CABG}-\mathrm{CPB}$ might be worried by evidence that surgeons perform fewer grafts with OPCAB compared with $\mathrm{CABG}-$ CPB. However, the absolute difference reported by a systematic review of 22 RCTs was only 0.2 grafts fewer with OPCAB. ${ }^{1}$ This magnitude of difference was also observed in the BHACAS trials and clearly had no effect on any outcome.

We observed a significant reduction in the odds of graft occlusion in BHACAS 2 compared with BHACAS 1, despite broader eligibility criteria. Although the interaction of trial by operation type was not statistically significant, we attribute this at least in part to the learning curve and evolution of OPCAB in our center during the trials, in particular the attention given to careful surgical technique with better stabilization and hemodynamic control. ${ }^{23}$ Aspects of techniques developed for OPCAB might also have led to improved $\mathrm{CABG}-\mathrm{CPB}$ technique.

\section{CONCLUSIONS}

Long-term health outcomes with OPCAB are similar to those with $\mathrm{CABG}-\mathrm{CPB}$. We found no evidence of longterm harm associated with OPCAB.

We thank the participants in the BHACASs, particularly for their willingness to contribute information over such a long period of follow-up. 


\section{References}

1. Cheng DC, Bainbridge D, Martin JE, Novick RJ. Evidence based perioperative clinical outcomes research group. Does off-pump coronary artery bypass reduce mortality, morbidity, and resource utilization when compared with conventional coronary artery bypass? A meta-analysis of randomized trials. Anesthesiology. 2005;102:188-203

2. Wijeysundera DN, Beattie WS, Djaiani G, Rao V, Borger MA, Karkouti K, et al. Off pump coronary artery surgery for reducing mortality and morbidity: meta-analysis of randomized and observational studies. J Am Coll Cardiol. 2005;46:872-82.

3. Ascione R, Lloyd CT, Underwood MJ, Lotto AA, Pitsis AA, Angelini GD. Economic outcome of off-pump coronary artery bypass surgery: a prospective randomized study. Ann Thorac Surg. 1999;68:2237-42.

4. Puskas JD, Williams WH, Mahoney EM, Huber PR, Block PC, Duke PG, et al. Off-pump vs. conventional coronary artery bypass grafting: early and 1-year graft patency, cost, and quality-of-life outcomes: a randomized trial. JAMA. 2004;291: 1841-9.

5. Nathoe HM, van Dijk D, Jansen EWL, Suyker WJ, Diephuis JC, van Boven WJ, et al. A comparison of on-pump and off-pump coronary bypass surgery in lowrisk patients. $N$ Engl J Med. 2003;348:394-402.

6. Cleveland JC Jr, Shroyer AL, Chen AY, Peterson E, Grover FL. Off-pump coronary artery bypass grafting decreases risk-adjusted mortality and morbidity. Ann Thorac Surg. 2001;72:1282-8.

7. Keogh BE, Kinsman R. Fifth National Adult Cardiac Surgical Database report for 2003. Henley-on-Thames, United Kingdom: Dendrite Clinical Systems; 2004.

8. Lim E, Drain A, Davies W, Edmonds L, Rosengard BR. A systematic review of randomized trials comparing revascularization rate and graft patency of off-pump and conventional coronary surgery. J Thorac Cardiovasc Surg. 2006;132: 1409-13.

9. Angelini GD, Taylor FC, Reeves BC, Ascione R. Early and midterm outcome after off-pump and on-pump surgery in Beating Heart Against Cardioplegic Arrest Studies (BHACAS 1 and 2): a pooled analysis of two randomised controlled trials. Lancet. 2002;359:1194-9.

10. Ascione R, Reeves BC, Taylor FC, Seehra HK, Angelini GD. Beating Heart Against Cardioplegic Arrest Studies (BHACAS 1 and 2): quality of life at mid-term follow-up in two randomised controlled trials. Eur Heart J. 2004; 25:765-70.

11. Schlosser T, Konorza T, Hunold P, Kühl H, Schmermund A, Barkhausen J. Noninvasive visualization of coronary artery bypass grafts using 16-detector row computed tomography. J Am Coll Cardiol. 2004;44:1224-9.

12. Ascione R, Caputo M, Calori G, Lloyd CT, Underwood MJ, Angelini GD. Predictors of atrial fibrillation after conventional and beating heart coronary surgery: a prospective randomised study. Circulation. 2000;102:1530-5.

13. Austen WG, Edwards JE, Frye RL, Gensini GG, Gott VL, Griffith LS, et al. A reporting system on patients evaluated for coronary artery disease. Report of the Ad Hoc Committee for Grading of Coronary Artery Disease, Council on Cardiovascular Surgery, American Heart Association. Circulation. 1975;51(suppl):5-40.

14. Taylor FC, Reeves BC, Ascione R, Angelini GD. Accuracy of reporting cardiac related events during long-term follow-up. Heart. 2003;89:331-2.

15. Spertus JA, Winder JA, Dewhurst TA, Deyo RA, Prodzinski J, McDonell M, et al. Development and evaluation of the Seattle Angina Questionnaire: A new functional status measure for coronary artery disease. J Am Coll Cardiol. 1995;25: 333-41.

16. Schroter S, Lamping DL. Coronary revascularisation outcome questionnaire (CROQ): development and validation of a new, patient based measure of outcome in coronary bypass surgery and angioplasty. Heart. 2004;90:1460-6.

17. Ware JE, Sherbourne CD. The MOS 36-item Short-Form Health Survey (SF-36): I. Conceptual framework and item selection. Med Care. 1992;30:473-83.

18. Ware JE, Kosinski M, Gandek B. SF-36 Health Survey. Manual and interpretation guide. Lincoln, RI: QualityMetric Incorporated; 2000.

19. EuroQol Group. EuroQol-a new facility for the measurement of health-related quality of life. Health Policy. 1990;16:119-208.

20. Widimsky P, Straka Z, Stros P, Jirasek K, Dvorak J, Votava J, et al. One-year coronary bypass graft patency: a randomized comparison between off-pump and on-pump surgery angiographic results of the PRAGUE-4 trial. Circulation. 2004; 110:3418-23.

21. Khan NE, De Souza A, Mister R, Flather M, Clague J, Davies S, et al. A randomized comparison of off-pump and on-pump multivessel coronary-artery bypass surgery. N Engl J Med. 2004;350:21-8.

22. Van Dijk D, Jansen EW, Hijman R, Nierich AP, Diephuis JC, Moons KG, et al. Cognitive outcome after off-pump and on-pump coronary artery bypass graft surgery: a randomized trial. JAMA. 2002;287:1405-12.
23. Watters MPR, Ascione R, Ryder IG, Ciulli F, Pitsis AA, Angelini GD. Haemodynamic changes during beating heart coronary surgery with the "Bristol technique." Eur J Cardiothorac Surg. 2001;19:34-40.

\section{Discussion}

Dr Soichiro Kitamura (Osaka, Japan). I thank the Association for the privilege of discussing this fine article. I also thank Professor Angelini for allowing me to read the manuscript before the presentation. My compliments on a well—designed and well—conducted clinical study demonstrating again that OPCAB can offer good quality of surgical intervention in terms of 6 to 8 years' graft patency, survival and survival free from MACEs, and patients' HRQoL after surgical intervention in comparison with conventional on-pump CABG.

This article is certainly the extension of a previously published randomized clinical trial called the BHACAS 1 and 2 conducted almost 10 years ago, and today, the chief researchers again demonstrated that OPCAB, as compared with on-pump CABG, can yield a similar level of long-term survival and quality of life based on the similar long-term graft patency evaluated by using 16-array MDCTA.

Your group reported in 2002 and 2004 that OPCAB provided significant reduction in postoperative morbidity compared with that seen after conventional on-pump CABG, such as a $25 \%$ reduction in postoperative atrial fibrillation, a $31 \%$ reduction in blood transfusion, fewer chest infections, shorter intensive care unit stay, and so on. Also, a midterm follow-up study showed that randomized patients had a similar generic and disease-specific quality of life. However, OPCAB remains in only $15 \%$ to $20 \%$ of total practice in Western countries, you said.

On the contrary, in Japan surgeons have adopted this technique for about $60 \%$ of patients undergoing isolated CABG, and in my institution, the National Cardiovascular Center, $98 \%$ of isolated CABG procedures have been performed using the OPCAB technique, probably because in Japan angiographic follow-up is rather routine, which can allow surgeons and cardiologists to evaluate and confirm their own results. Very interestingly, once surgeons are trained and accustomed to do it, they are reluctant to go back to on-pump CABG because they are more comfortable with this technique because of easy hemostasis and easy adjustment of the graft length for complicated graft arrangement. I believe the basic reason for this technology not being popularized well, regardless of less use of resources and reduction of early postoperative morbidity, is the surgeons' attitude anesthesiologists' attitude, or both and the training system.

My first question is this: Why has reduction in postoperative morbidity contributed very little to the wide application of this technique? You mentioned that this was probably because of the concern about long-term graft patency and clinical results. What would you think about the importance of a training system, change of attitude, or both for OPCAB rather than evidence demonstrated by a handful of surgeons who are used to doing it?

Dr Angelini. Thank you, Dr Kitamura, for your kind remarks and your very appropriate question, which is not easy to answer. I have visited Japan on many occasions, and I have always been surprised by the skill and level with which you have adopted OP$\mathrm{CAB}$ surgery and also arterial revascularization, 2 techniques that are very poorly used in the Western world. 
I think we have plenty of evidence on the benefits of OPCAB surgery. To say, as I heard this morning, that a reduction in blood loss is not such important evidence is a very feeble excuse. I think this is a technique that requires an institution's commitment to it and not just the surgeon and anesthetist but the whole team, and you have to be prepared to go through a learning process, which can be painful. Perhaps this is one of the reasons why surgeons are not prepared to adopt OPCAB surgery.

Maybe there is another explanation. I was reading an editorial written 5 or 6 years ago by Lawrence Bonchek, a very well-known, now retired, American surgeon, who asked, "Is off-pump for everybody?" After all, not all of us can perform mitral valve repair, extensive arterial revascularization, or aortic valve sparing. Therefore perhaps we should see the off-pump procedure as a specialized procedure and to think that everybody will be able to adopt it is perhaps just not feasible.

Dr Kitamura. Well, if I am correct, you are planning to conduct a new randomized trial of OPCAB versus $C A B G-C P B$ in patients with poor left ventricular function. Conversion from OPCAB to on-pump CABG in a hasty situation has been reported to result in high mortality. Urgent conversion occurs more often in patients with poor and large left ventricles. At present, would you think OPCAB should be limited for the patients with a low probability of conversion until new evidence comes out?

Dr Angelini. Thank you, again, for this question. We are indeed going to carry out a large study supported by the Medical Research Council in the United Kingdom. The plan is to enroll 5000 patients (EuroSCORE $>5$ ). Therefore these will be patients with poor ventricular function but also redo operations, patients with renal impairment, lung dysfunction, and so forth.

As far as conversion is concerned, our experience was reported about 2 years ago in the European Journal of Cardio-Thoracic Surgery; for the period 1995 to 2005 , we had an overall conversion of $1.1 \%$, going from $5.2 \%$ in the 1995 to 1996 period to less than $0.4 \%$ in the most recent years. I think in institutions in which OPCAB surgery has been adopted, after a proper learning curve, the risk of conversion is very small.

Dr Kitamura. I once again congratulate you and your associates on this excellent clinical research, and I hope this evidence can significantly contribute to the prevalence of the OPCAB technique. Thank you very much. 


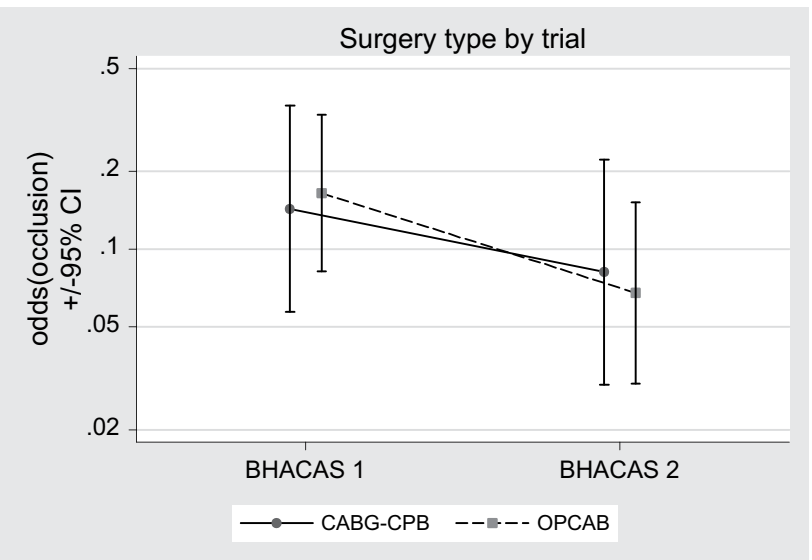

FIGURE E1. Graphic representation of interaction of operation type by trial. Error bars are 95\% confidence intervals. For the purposes of the graph, the reference stratum is as follows: distal segment, saphenous vein, and inferior territory. BHACAS, Beating Heart Against Cardioplegic Arrest Studies; $C A B G-C P B$, Coronary artery bypass grafting with cardiopulmonary bypass; $O P C A B$, off-pump coronary artery bypass. 


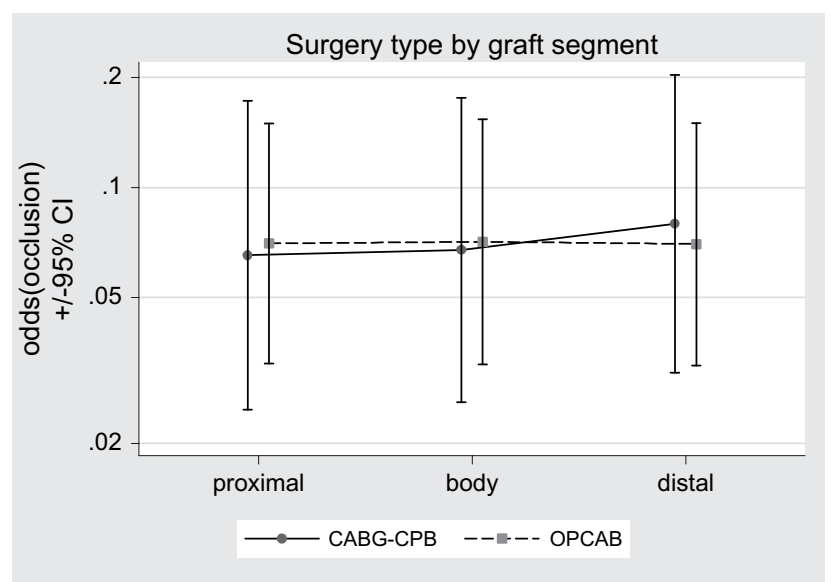

FIGURE E2. Graphic representation of operation type by graft segment. Error bars are $95 \%$ confidence intervals. For the purposes of the graph, the reference stratum is as follows: Beating Heart Against Cardioplegic Arrest Studies 2, saphenous vein, and inferior territory. $C A B G-C P B$, Coronary artery bypass grafting with cardiopulmonary bypass; $O P C A B$, off-pump coronary artery bypass. 
TABLE E1. Logistic regression of graft occlusion: Model details without interaction of operation type by trial

\begin{tabular}{|c|c|c|c|c|c|}
\hline Regression term & Odds ratio & Robust standard error & $\mathbf{z}$ & $\boldsymbol{P}>|\mathbf{z}|$ & $95 \%$ Confidence interval \\
\hline CABG-CPB & 1.00 & & & & \\
\hline OPCAB & 0.998989 & 0.3019464 & -0.00 & .997 & $0.5524377-1.806501$ \\
\hline BHACAS 1 & 1.00 & & & & \\
\hline BHACAS 2 & 0.488524 & 0.149528 & -2.34 & .019 & $0.2681327-0.8900672$ \\
\hline Proximal segment & 1.00 & & & & \\
\hline Body segment & 1.022302 & 0.0561426 & 0.40 & .688 & $0.9179798-1.13848$ \\
\hline Distal segment & 1.105597 & 0.0476357 & 2.33 & .020 & $1.016067-1.203017$ \\
\hline ITA* & 1.00 & & & & \\
\hline Radial artery & 2.725595 & 2.131022 & 1.28 & .200 & $0.5887685-12.61764$ \\
\hline Saphenous vein & 1.338772 & 0.5262213 & 0.74 & .458 & $0.6196282-2.892559$ \\
\hline Inferior & 1.00 & & & & \\
\hline Anterior & 1.856419 & 0.8938422 & 1.28 & .199 & $0.7224922-4.770004$ \\
\hline Lateral & 1.573124 & 0.6195576 & 1.15 & .250 & $0.7269872-3.404077$ \\
\hline
\end{tabular}

Logistic regression

Number of observations $=1364$

Wald $\chi^{2}(8)=18.41$

Probability $>\chi^{2}=0.0184$

Log pseudolikelihood $=-459.49033$

Pseudo $R^{2}=0.0362$

(Standard error adjusted for 199 clusters in id)

$C A B G-C P B$, Coronary artery bypass grafting with cardiopulmonary bypass; $O P C A B$, off-pump coronary artery bypass; $B H A C A S$, Beating Heart Against Cardioplegic Arrest Studies; ITA, internal thoracic artery. *Nineteen free internal thoracic artery grafts, none of which were occluded, were pooled with pedicle internal thoracic artery grafts so that they could be included in the model. 
TABLE E2. Logistic regression of graft occlusion: Model details with interaction of operation type by trial

\begin{tabular}{|c|c|c|c|c|c|}
\hline Regression term & Odds ratio & Robust standard error & $\mathbf{z}$ & $\boldsymbol{P}>|\mathbf{z}|$ & $\mathbf{9 5} \%$ Confidence interval \\
\hline CABG-CPB & 1.00 & & & & \\
\hline OPCAB & 1.148089 & 0.4513583 & 0.35 & .725 & $0.5312944-2.480938$ \\
\hline BHACAS 1 & 1.00 & & & & \\
\hline BHACAS 2 & 0.5685687 & 0.247001 & -1.30 & .194 & $0.2426604-1.332192$ \\
\hline OPCAB $\times$ BHACAS 2 & 0.7233592 & 0.4255386 & -0.55 & $.582 \dagger$ & $0.2283537-2.291394$ \\
\hline Proximal segment & 1.00 & & & & \\
\hline Body segment & 1.019942 & 0.0562281 & 0.36 & .720 & $0.9154825-1.136322$ \\
\hline Distal segment & 1.104232 & 0.0476268 & 2.30 & .022 & $1.014722-1.201638$ \\
\hline ITA* & 1.00 & & & & \\
\hline Radial artery & 2.696345 & 2.08055 & 1.29 & .199 & $0.5942548-12.23428$ \\
\hline Saphenous vein & 1.341522 & 0.5267276 & 0.75 & .454 & $0.6214223-2.896068$ \\
\hline Inferior & 1.00 & & & & \\
\hline Anterior & 1.884035 & 0.9009327 & 1.32 & .185 & $0.7379899-4.809808$ \\
\hline Lateral & 1.558439 & 0.6153068 & 1.12 & .261 & $0.7188139-3.378807$ \\
\hline
\end{tabular}

Logistic regression

Number of observations $=1364$

Wald $\chi^{2}(9)=18.87$

Probability $>\chi^{2}=.0263$

Log pseudolikelihood $=-459.07974$

Pseudo $R^{2}=0.0370$

(Standard error adjusted for 199 clusters in id)

$C A B G-C P B$, Coronary artery bypass grafting with cardiopulmonary bypass; $O P C A B$, off-pump coronary artery bypass; $B H A C A S$, Beating Heart Against Cardioplegic Arrest Studies; ITA, internal thoracic artery. *Nineteen free internal thoracic artery grafts, none of which were occluded, were pooled with pedicle internal thoracic artery grafts so that they could be included in the model. $\dagger$ The highlighted $P$ value refers to the interaction of interest $\left(\chi^{2}=0.30, d f=1, P=.582\right)$. 
TABLE E3. Logistic regression of graft occlusion: Model details with interaction of operation type by graft segment

\begin{tabular}{|c|c|c|c|c|c|}
\hline Regression term & Odds ratio & Robust standard error & $\mathbf{Z}$ & $\boldsymbol{P}>|\mathbf{z}|$ & 95\% Confidence interval \\
\hline $\mathrm{CABG}-\mathrm{CPB}$ & 1.00 & & & & \\
\hline OPCAB & 1.075826 & 0.3407526 & 0.23 & .818 & $0.5782771-2.001465$ \\
\hline BHACAS 1 & 1.00 & & & & \\
\hline BHACAS 2 & 0.4879398 & 0.1495132 & -2.34 & .019 & $0.2676353-0.8895883$ \\
\hline Proximal segment & 1.00 & & & & \\
\hline Body segment & 1.031752 & 0.0541339 & 0.60 & .551 & $0.9309244-1.1435$ \\
\hline Distal segment & 1.218846 & 0.0928309 & 2.60 & .009 & $1.04983-1.415073$ \\
\hline OPCAB $\times$ body & 0.9800255 & 0.1091933 & -0.18 & $.036 \dagger$ & $0.7877665-1.219207$ \\
\hline $\mathrm{OPCAB} \times$ dist & 0.8174873 & 0.0702114 & -2.35 & $0.6908345-0.9673597$ & \\
\hline $\mathrm{ITA}^{*}$ & 1.00 & & & & \\
\hline Radial artery & 2.737529 & 2.138362 & 1.29 & .197 & $0.5921897-12.65484$ \\
\hline Saphenous vein & 1.337091 & 0.5256787 & 0.74 & .460 & $0.6187425-2.889427$ \\
\hline Inferior & 1.00 & & & & \\
\hline Anterior & 1.861796 & 0.8972326 & 1.29 & .197 & $0.723974-4.787858$ \\
\hline Lateral & 1.574507 & 0.6204827 & 1.15 & .249 & $0.7272819-3.408683$ \\
\hline
\end{tabular}

Logistic regression

Number of observations $=1364$

Wald $\chi^{2}(10)=21.19$

Probability $>\chi^{2}=0.0198$

Log pseudolikelihood $=-459.35623$

Pseudo $R^{2}=0.0364$

(Standard error adjusted for 199 clusters in id)

$C A B G-C P B$, Coronary artery bypass grafting with cardiopulmonary bypass; OPCAB, off-pump coronary artery bypass; BHACAS, Beating Heart Against Cardioplegic Arrest Studies; ITA, internal thoracic artery. ${ }^{*}$ Nineteen free internal thoracic artery grafts, none of which were occluded, were pooled with pedicle internal thoracic artery grafts so that they could be included in the model. $\dagger$ The highlighted $P$ value refers to the overall interaction term $\left(\chi^{2}=6.65, d f=2, P=.036\right)$. 